\title{
Juegos digitales desde el punto de vista de los profesores. Una experiencia didáctica en aulas primaria catalanas
}

\section{Digital games from teachers' point of view. A didactic experience in Catalan Primary classrooms}

\begin{abstract}
José Luis Eguia-Gómez
Universitat Politècnica

de Catalunya, España

eguia@ege.upc.edu
\end{abstract}

Fecha de recepción: 05-02-2015

Fecha de revisión: 26-03-2015

\section{Palabras clave:}

Juegos digitales; juegos serios; aprendizaje; profesores de primaria; punto de vista de profesores; innovación educativa.

\section{Resumen}

Ruth Contreras

Universitat de Vic-

Universitat Central de

Catalunya, España

ruth.contreras@uvic.cat

\begin{abstract}
Lluis Solano
Universitat Politècnica

de Catalunya, España

solano@lsi.upc.edu
\end{abstract}

Fecha de aceptación: 11-05-2015 Fecha de publicación: 15-06-2015

Keywords:

Digital games; serious games; learning; primary teachers; teachers' point of view; educational innovation.

Abstract

Este artículo presenta una experiencia

This article presents a didactic experience didáctica centrada en la aplicación de un juego digital en las aulas catalanas de quinto y sexto grado de educación primaria. En él se explica y analizan los efectos de su aplicación en la práctica docente, tomando el punto de vista de los docentes que participaron de la experiencia. El documento se basa en el juego digital llamado Miquel Crusafont, un juego que cuenta con el apoyo de la Generalitat de Catalunya. El estudio obtiene las opiniones de un grupo seleccionado de once profesores -con edades entre 24 y 52 en relación a dicha innovación metodológica. Se llevaron acabo entrevistas con el fin de obtener el punto de vista del profesorado. Los resultados muestran que los educadores aprecian la potencialidad del juego digital for applying a digital game in Catalan classrooms of grades fifth and sixth of Primary Education. It explains and analyzes the effects of its implementation on the educational practice, taking the teachers' viewpoint who participated in the experience. The paper is based on the digital game called Miquel Crusafont, a digital game supported by Generalitat de Catalunya. The study tries to understand the sense that such methodological innovation made for the selected group of eleven primary school teachers -of ages 24 to $52-$. Interviews were done in order to see the teacher's behaviours and to know their point of view. The results pointed that the teachers appreciated the potentiality of the digital game to develop 
para desarrollar diversas habilidades a través de áreas como las matemáticas y consideran la forma en como pueden alentar a los estudiantes a realizar algunas actividades de aprendizaje colaborativo. Señalaron, además, algunas dificultades para utilizar un juego digital de estas características en las aulas: falta un tiempo destinado específicamente a ello en el plan de estudios, algunos juegos en el mercado no son apropiados para la enseñanza o no cuentan con un manual de uso que muestre la potencialidad del juego

\section{Introducción}

Diversas investigaciones podrían explicar la validez del uso de un juego digital o juego serio en la educación desde una perspectiva pedagógica. La primera relación podría explicarse con el estudio de los procesos cognitivos y no solo con el estudio de los resultados. La aplicación de contenidos de un curso se ajusta a un proceso de construcción del conocimiento que sirve como una doble estimulación para lograr un proceso de mejora del aprendizaje y del resultado (Vygotsky, 1978). Otros autores mencionan que mediante los juegos digitales es posible el desarrollo de habilidades sociales (Perrota et al, 2013), la motivación hacia el aprendizaje (Kenny y McDaniel, 2011), una mejora en la atención, la concentración, el pensamiento complejo y la planificación estratégica (Kirriemuir y McFarlane, 2004). Incluso se explica que ayudan en conocimientos diverse Math skills and encourage some collaborative learning activities. They also identified difficulties that obstructed the use of a digital game in classrooms: lack of time in the curriculum and some games in the market are not appropriate to teach or they do not have a user's guide showing all the potentiality of the game.

multidisciplinarios (Mitchell y Savill-Smith, 2004), al pensamiento lógico y crítico y a mejorar habilidades que ayudan a resolver diversos problemas (Higgins, Grant y Shah, 1999), desarrollar habilidades cognitivas y en la toma de decisiones técnicas (Bonk y Dennen, 2005). El profesor puede desplegar diferentes estrategias orientadas a potenciar el conocimiento de hechos y datos concretos, gracias a los contenidos educativos presentes en el juego como, por ejemplo, conocer la vida de un personaje y su actividad, y el conocimiento condicional (Paris, Lipson y Wixson, 1983), utilizando conocimientos adquiridos previamente, para plantear estrategias de forma consciente. En teoría, la investigación longitudinal en aprendizaje basado en juegos (en adelante ABJ) y en juegos serios (JS) ofrece la posibilidad de variar el contexto, las variables de intermediación, 
mejorar la motivación de los estudiantes, así como la experiencia de los facilitadores del juego, y una mejora en las instalaciones institucionales (Kenny y McDaniel, 2011). En el contexto catalán los estudios que encontramos en este campo son limitados, pero existe un uso de juegos digitales en las aulas. Según el estudio European Schoolnet realizado entre octubre de 2008 y febrero del 2009, que pretende identificar prácticas de profesores utilizando juegos digitales, en Cataluña existe un mayor uso de juegos digitales en las aulas en comparación con países como Francia, Italia, Dinamarca o Austria (Kearney, 2011). Sin embargo este estudio no proporciona datos específicos sobre el uso de los mismos en las aulas catalanas. Por otro lado se cuenta con iniciativas como la red Telemática Educativa de Cataluña (XTEC), que tiene la función de proporcionar una gran variedad de contenidos -entre los que se incluyen juegos digitales-, propuestas y servicios específicos disponibles en Internet para el sector educativo. Con estos recursos, los profesores deben buscar una correspondencia adecuada entre las tareas didácticas y el uso de juegos (Wu y Wang, 2012), como una forma de aplicar una doble estimulación para lograr un proceso de mejora del aprendizaje y del resultado como menciona Vygotsky. Y si el juego seleccionado entra en conflicto con las tareas asignadas al alumnado, el profesor puede reconsiderar cambiar las tareas. El estímulo puede soportar la actividad de aprendizaje para ambos métodos: la correspondencia entre las tareas escolares y el uso de juegos. Los profesores deben darse cuenta de esto y analizar cuáles son las mejores herramientas para cumplir los objetivos de un curso y así estimular el aprendizaje en los estudiantes cuando sea necesario (Wu y Wang, 2012).

En esta investigación se analizan e interpretan los efectos en la práctica educativa de la introducción de un juego digital que sirve de soporte en las materias de quinto $y$ sexto curso de Educación Primaria. Se ha tomando como referencia el punto de vista del profesorado. El informe se ha dividido en dos partes. En la primera se describe el diseño y desarrollo de la propuesta didáctica implementada, explicitando los objetivos del juego, sus fundamentos pedagógicos, así como la propuesta de evaluación de las actividades realizadas. En la segunda parte, se analizan los resultados de las entrevistas realizadas a los profesores. Indagar en los objetivos de nuestra propuesta y en la concepción global que se formó el profesorado gracias a la experiencia constituye el núcleo principal de este trabajo. 


\section{El juego digital como una experiencia didáctica}

\subsection{Descripción y metodología}

El propósito de la experiencia fue crear un juego digital que sirviera de apoyo a la docencia presencial. El juego denominado Miquel Crusafont ha sido financiado por la Generalitat de Catalunya y se centra en dar a conocer el personaje, situarlo en la jus historia relacionando los hechos ocurridos contemporáneos a él, y dar a conocer su legado, sus estudios y su trabajo en paleontología. El área básica curricular que se trabaja es el conocimiento del medio natural y social. Otras áreas curriculares relacionadas son las matemáticas, la lengua catalana e inglesa y la educación artística. Como se trata de un juego para ordenador, el ámbito de trabajo puede realizarse en casa o bien en al aula durante varias sesiones. Paralelamente el profesorado puede trabajar diversos aspectos del currículo. Los objetivos son: 1) facilitar el aprendizaje aplicando las posibilidades didácticas de un juego digital; 2) integrar el ABJ o los JS en un contexto curricular innovando metodológicamente el tratamiento de contenidos de quinto y sexto curso de primaria.

La propuesta didáctica asume los principios del constructivismo, los juegos permiten al jugador construir su propia comprensión del mundo. El juego es una actividad libre que se realiza sin una obligación externa, y esta actividad incentiva al alumnado a experimentar, a probar múltiples soluciones, descubrir la información y nuevos conocimientos sin temor a equivocarse. Se prima así una metodología basada en la experimentación, la autonomía y la autoevaluación, que busca: a) favorecer la motivación; b) promover su compromiso con el aprendizaje; c) activar conocimientos previos; d) motivar la exploración activa; e) promover la colaboración como medio para facilitar el aprendizaje. En definitiva, el juego se concibe como un espacio que amplía y complementa el trabajo presencial del profesor.

\subsection{Descripción y metodología}

\subsubsection{Aulas de clase seleccionadas y perfil de los participantes}

La experiencia se realizó en dos escuelas durante el año 2012. Los escenarios fueron la ubicadas en la provincia de Barcelona Escola Nostra Llar de la ciudad de Sabadell 
y el Centro de Educación Infantil y Primaria Octavio Paz de la ciudad de Barcelona. Ambos centros recomendados por el Centro de Recursos Pedagógicos Les Corts. Tras sopesar lo que podría significar introducir una nueva actividad en el curso, se optó por sugerir el uso del juego en dos momentos específicos, y durante dos dias. Se escogió para ello a un grupo de educadores con las siguientes características:

- Profesorado de quinto y sexto curso de primaria, con edad en un rango de 24 a 52 años.

- Entre los profesores que participaron había un número mayor de mujeres (9) que de hombres (3).

- Se encuentran a favor de la inclusión de juegos digitales en el aula (6) pero tambien se muestran reacios a su uso (6).

- Están familiarizados con herramientas multimedia disponibles en la red Telemática Educativa de Cataluña (XTEC) donde pueden encontrarse algunos juegos digitales.

- Hacen uso tanto de Internet como de otros dispositivos, como tabletas digitales o teléfonos móviles a nivel personal.

- Consideran positivo el lenguaje audiovisual y la interacción para atraer la atención de los niños.

\subsection{El juego digital utilizado}

\subsubsection{Aulas de clase seleccionadas y perfil de los participantes}

El juego está estructurado en 14 niveles, y se basa en secuencias temporales, de modo que cada bloque puede contener eventos que ocurren en diferentes espacios y tiene una serie de objetivos que los jugadores deben superar, aumentando la dificultad en cada nivel. La figura 1 muestra el mapa de niveles. La animación inicial dispuesta en el juego sirve de presentación para situar al jugador en el espacio físico y temporal donde se desarrolla la historia. Así, el juego comienza en la estación de Francia de Barcelona, donde Sandra Li -el avatar-, seguirá los pasos de Crusafont. Su primer contacto con la ciencia despertará su deseo de conocimiento y esto la empujará a emprender un viaje para conocer la vida del personaje, el mundo de la paleontología y los hechos más relevantes

\begin{tabular}{|c|c|c|}
\hline & \multicolumn{2}{|l|}{ Mundo real } \\
\hline & Presentación & \\
\hline Nivel 1 & Estación de Francia & \\
\hline Nivel 2 & Trabajo de campo & \\
\hline Nivel 3 & Laboratorio & \\
\hline Nivel 4 & Mund & naginar \\
\hline Nivel 5 & Exposición universal & \\
\hline Nivel 6 & La creación del museo & \\
\hline Nivel 7 & Las excavaciones & \\
\hline Nivel 8 & La guerra civil & \\
\hline Nivel 9 & El jardin botánico & \\
\hline Nivel 10 & Divulgación cientifica & \\
\hline Nivel 11 & $\frac{1}{\text { La carrera espacial }}$ & \\
\hline Nivel 12 & $\frac{1}{\text { los Beatles }}$ & \\
\hline Nivel 13 & Final & \\
\hline
\end{tabular}

Figura 1: Mapa de niveles. 
de la historia del siglo XX. En este nivel se recuerda, de manera muy simple, el cálculo de fracciones $y$ se hace referencia al modernismo. Durante el nivel 2, Sandra conocerá a Miquel, de quien aprenderá el método científico: observación, hipótesis, experimentación, conclusión y elaboración de una teoría. Por eso, después de observar cómo recoge muestras sobre el terreno ella podrá reunir las propias para ir al laboratorio. En este espacio Sandra podrá experimentar con los huesos y fósiles reunidos para formar esqueletos de varios animales; esto le permitirá adentrarse en el "mundo imaginario", el siguiente nivel donde simulará la vida de estos animales. El uso de un imaginario que contenga información relevante del juego se utiliza para atraer a los alumnos a través de lo irreal, y constituye un espacio paralelo al

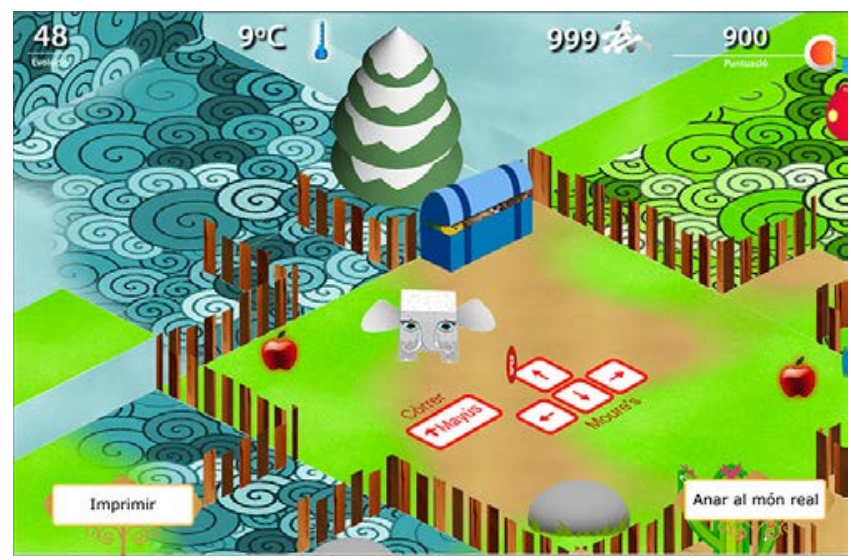

Figura 2. Nivel 4, "Mundo imaginario".

Esto da lugar a un espacio que incentiva la creatividad. La característica principal del mundo imaginario, (figura 2), es que los contenidos forman parte de las reglas de juego y tanto elementos como personajes se aprecian en vista tridimensional. Por ejemplo, la supervivencia o evolución de las especies está incluida en las reglas, es decir, a medida que el alumno juega, el animal puede evolucionar. Con ello el jugador aprenderá conceptos relativos a la evolución, el clima y la geografía.

En el mundo imaginario se estudiarán cuatro hábitats: la montaña, el humedal, el desierto y el bosque húmedo. Y a medida que evolucionan los animales, el jugador podrá tener la posibilidad de imprimirlos en papel. Fuller y Jenkins (1995) mencionan que los videojuegos ofrecen historias espaciales y que son espacios con un alto potencial narrativo. Los jugadores pueden imprimir y jugar con los avatares y crear así una extensión del juego virtual. Figura 3.
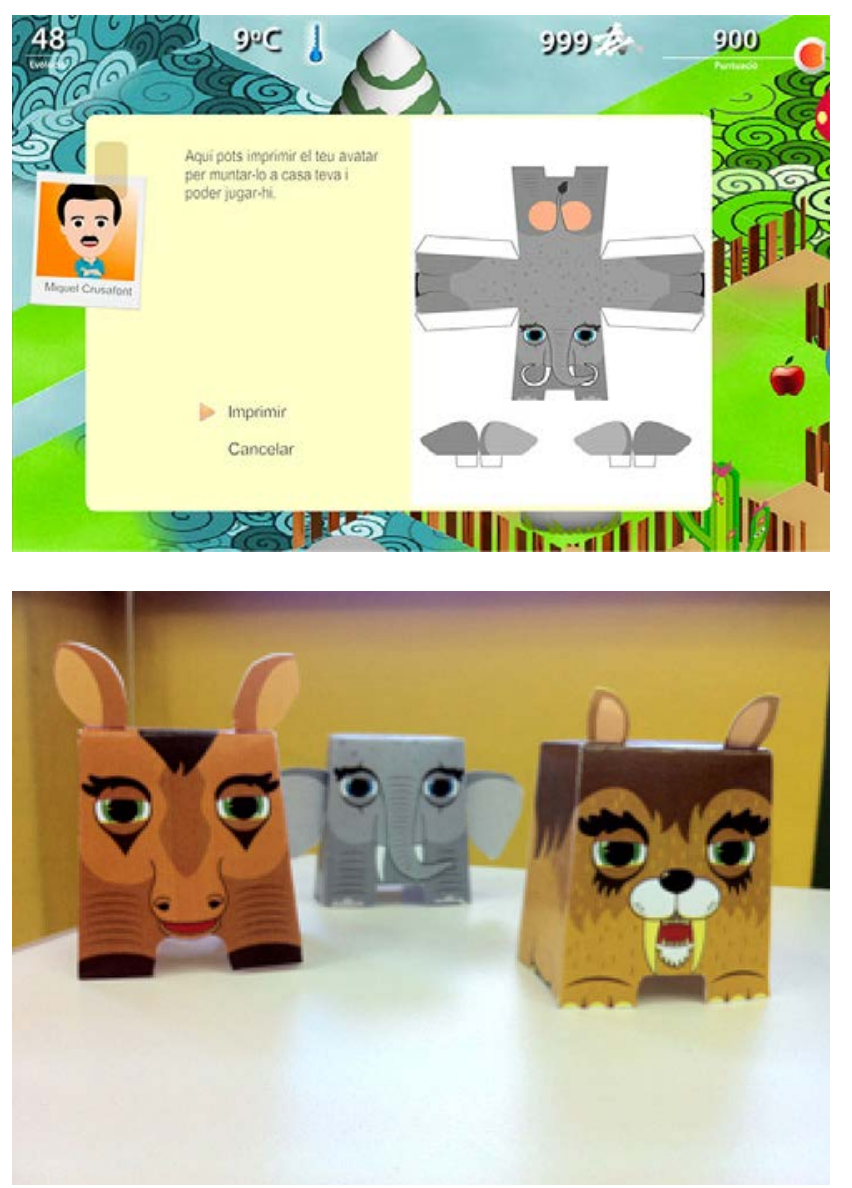

Figura 3. Personajes de Miquel Crusafont en papel. 
En el nivel 5, Sandra paseará por la exposición universal de Barcelona y descubrirá el Novecentismo, la corriente literaria y artística catalana del final del siglo XIX y el inicio del XX. En el siguiente nivel se plantearán cuestiones históricas, como la inauguración de un museo con su sección de paleontología a cargo de Crusafont. También se hace énfasis en figuras geométricas básicas, que se pueden aprovechar para trabajar en el aula. En el siguiente nivel, Sandra tiene que recoger muestras de huesos de las excavaciones paleontológicas. En este momento, justo en medio de la Guerra Civil, en el nivel 8, la alegoría del dolor y el sufrimiento de la guerra se verán reflejados en el cuadro que Pablo Picasso pintó: el Gernika. Esto dará paso a una transición, donde Sandra tendrá que pasear por un jardín botánico. Durante el recorrido encontrará información y constatará la importancia de la interdisciplinariedad del conocimiento científico, puesto que la botánica permite hacer avances en la paleontología. En el nivel 10, Sandra realizará un viaje al pasado a la época de los dinosaurios. Más tarde se hará incidencia en la importancia de los viajes espaciales para el progreso de la ciencia y la técnica. Ahí Sandra, ya en el nivel 11, tendrá que soportar los entrenamientos de los astronautas practicando su inglés.

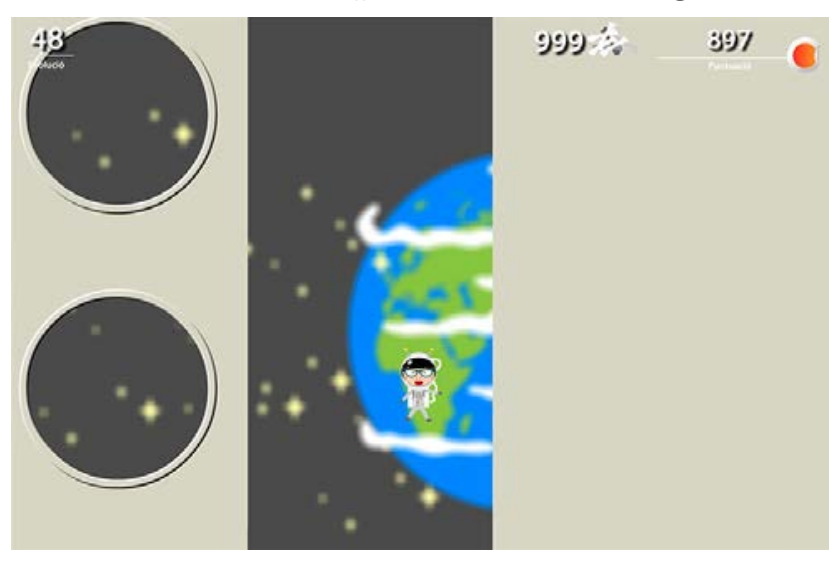
Figura 4. Nivel 11, "La Carrera Espacial"

Uno de los hechos más relevantes en cuanto a la cultura y los movimientos sociales contemporáneos a escala mundial fue la irrupción de la música de los Beatles. En julio de 1965 el grupo tocó en la plaza Monumental de Barcelona. En este nivel Sandra tendrá que encontrar al grupo inglés. De esta forma se llega al nivel final, donde se mostrará una síntesis de los hechos más relevantes relacionados con la vida y la historia de Crusafont.

\subsubsection{Competencias básicas en el currículo de educación primaria}

El juego tiene por objetivo desarrollar competencias básicas. En este sentido, el tratamiento de las competencias es el siguiente:

a) Competencia artística y cultural (comunicativa). Es el conocimiento, la comprensión y la valoración crítica de diferentes manifestaciones culturales y artísticas, que se utilizan como fuente de enriquecimiento y se consideran parte del patrimonio de cada cultura. El juego destaca varias manifestaciones culturales y artísticas, como el modernismo en Cataluña al inicio del siglo XX, o la obra Gernika de Picasso, y las relaciona con el contexto histórico.

b) Tratamiento de la información y 
competencia digital (metodológica). Trata la captación, la selección, el registro y el procesamiento de información, con el uso de técnicas y estrategias diversas con una actitud crítica y reflexiva. Requiere el dominio de lenguajes específicos básicos (textual, numérico, icónico, visual, gráfico y sonoro). El alumnado interactua con el juego utilizando lenguaje audiovisual.

c) Competencia matemática (metodológica). Habilidad para comprender, utilizar y relacionar números y aspectos espaciales de la realidad. Incluye operaciones básicas, símbolos y el razonamiento matemático, así como problemas y situaciones relacionadas con la vida cotidiana, el conocimiento científico o el mundo laboral y social. En diferentes fases del juego el alumnado debe resolver, bien con operaciones o mediante razonamiento matemático, situaciones relacionadas con la vida cotidiana.

d) Competencia de aprender a aprender (metodológica). Habilidad para conducir el aprendizaje y ser capaz de continuar aprendiendo de manera eficaz y autónoma de acuerdo con objetivos y necesidades propias. En el juego se dan instrumentos para que se investigue más allá y se profundice en el conocimiento.

e) Competencia autonomía e iniciativa (personal). Adquisición de la conciencia y aplicación de un conjunto de valores y actitudes personales interrelacionadas, como la responsabilidad, la perseverancia, el conocimiento de sí mismo, la autoestima, la creatividad, la autocrítica, el control emocional, aprender de los errores y asumir riesgos. En el juego se presentan diversas situaciones en las que el alumnado tiene que tomar decisiones para seguir determinados caminos y continuar en los niveles.

f) Competencia de conocimiento e interacción con el mundo físico (convivir con el mundo y habitarlo). Movilización de saberes que tienen que permitir al alumnado comprender las relaciones que se establecen entre la sociedad y su entorno para hacer un uso responsable de los recursos naturales y cuidar el medio ambiente. Incluye también el desarrollo y aplicación del pensamiento científicotécnico para interpretar información, hacer predicciones y tomar decisiones.

g) Competencia social y ciudadana (convivir con el mundo y habitarlo). Capacidad para comprender la realidad social en que se vive, afrontar la convivencia y los conflictos empleando el juicio ético que se basa en los valores y las prácticas democráticas actuando con criterio propio. En el juego se ofrece una historia que permite comprender la realidad social en que se vive.

\subsubsection{Objetivos generales de la etapa de primaria}

El juego, dentro del marco del currículo de la de los siguientes objetivos de la etapa: educación primaria, quiere contribuir al logro • Tener conciencia del valor del trabajo 
individual y colectivo y desarrollar hábitos de esfuerzo y trabajo, así como actitudes de confianza, iniciativa personal, autodisciplina, sentido crítico y responsabilidad.

- Desarrollar competencias matemáticas básicas, iniciándose en la resolución de problemas que requieran la realización de operaciones elementales de cálculo, conocimientos geométricos y estimaciones.

- Conocer, valorar y estimar el entorno natural, social y cultural cercano, y la capacidad de extrapolar los conocimientos al mundo en general; comprender, a partir de la observación de hechos y fenómenos sencillos, los mecanismos principales que rigen el entorno para ser capaz de tomar compromisos.

- Aplicar, en contextos diversos, los diferentes conocimientos adquiridos $\mathrm{y}$ los recursos propios, con el objetivo de resolver problemas, situaciones personales y necesidades de la vida cotidiana.

Conocer y valorar el medio natural, así como los animales más cercanos al ser humano, y adoptar comportamientos para ayudar a protegerlos.

\subsubsection{Criterios de evaluación}

Una vez realizada la actividad del juego y con las actividades posteriores, o previas al aula, el profesorado puede valorar la posibilidad de evaluar el aprendizaje adquirido. Los criterios propuestos son los siguientes:

- Analizar elementos físicos y humanos, y los principales factores de cambio producidos por los procesos naturales y la actividad humana a lo largo del tiempo. Reconocer la diversidad de paisajes de Cataluña y España, así como la necesidad de preservar la riqueza patrimonial.

- Relacionar la estructura de un ser vivo con las funciones que realiza.

- Reconocer y respetar la diversidad de manifestaciones culturales del entorno.

- Identificar, describir y analizar procesos de cambio y transformación social, cultural, económica y tecnológica en el entorno.
- Utilizar diferentes tipos de fuentes documentales para obtener información sobre la sociedad de épocas pasadas y situar los hechos en líneas de tiempos.

En el área de las matemáticas:

- Cuantificar situaciones de la vida real como un aspecto que favorece la comparación, la ordenación y la clasificación.

- Reconocer y comprender los problemas, buscar y utilizar cifras y signos adecuados para seleccionar y organizar datos necesarios, estimar una respuesta razonable y desarrollar estrategias de resolución.

- Interpretar el sistema de numeración decimal; interpretar y utilizar los números naturales y fraccionarios, de acuerdo con contextos de la vida cotidiana.

- Utilizar el significado de las operaciones 
con los números naturales y fraccionarios de forma apropiada a cada contexto; desarrollar agilidad en el cálculo exacto y aproximado con operaciones básicas.

- Identificar, reconocer y describir con precisión figuras y cuerpos geométricos del entorno; clasificarlas de acuerdo con las características geométricas (vértices, ángulos, etc.).

El área de lengua catalana e inglesa se va tratando a lo largo del juego mediante la interacción con los textos que aparecen. Se trabaja mediante:

- La comprensión de la información escrita en alguno de los formatos posibles que hacen referencia a cualquier ámbito de la escuela y la vida cotidiana.

- La respuesta a preguntas a partir de un texto.

- Comprensión de mensajes cotidianos de intercambio social y de mensajes relacionados con el contenido temático, procedencia geográfica, etc.

- Comprender autónomamente textos escritos. Saber responder a preguntas relacionadas con los textos que se han leído.

- Comprender y extraer información relevante de textos.

\section{La experiencia Didáctica desde el punto de vista de los profesores}

\subsection{Diseño de la evaluación}

El propósito de la evaluación fue analizar y valorar la experiencia de los profesores. Antes de realizar las entrevistas, los profesores conocieron el juego y se solicitó que lo utilizasen en clase para observar el comportamiento de los alumnos. Cada profesor pudo comprobar la actitud, la colaboración y el estado de ánimo de los alumnos durante las sesiones. Observaron la actividad, participaron comentando el juego con los estudiantes y ayudaron en diversas actividades del juego. Durante la sesión se hicieron observaciones, y finalizada la actividad realizamos las entrevistas en profundidad. Esta se dividió en cuatro apartados, cada uno de los cuales incidía en una dimensión: circunstancias personales, actitud, dificultades, y recomendaciones. La construcción de las preguntas tenían como finalidad: 1) conocer sus circunstancias personales 2) identificar la actitud del profesor ante los juegos digitales en las aulas, 3) detectar las dificultades que presenta la introducción de un juego de este tipo en el aula, y 4) reunir el punto de vista personal del entrevistado en relación a cómo debería de ser un juego digital educativo. Creímos interesante añadir el punto dos, porque la actitud de los profesores es un aspecto esencial en el proceso de enseñanza, y sobre todo es 
básico cuando se aplican juegos digitales en un curso, mientras que en el tercer y cuarto punto se pretendía conocer un punto de vista personal con el fin de detectar estrategias orientadas a potenciar conocimientos, tal y como respalda Flavell (1985). En relación a las circunstancias personales, se reunieron

\subsection{Análisis de los resultados}

Durante la sesión se observó que los niños se adaptaron fácilmente al juego. Como jugadores, asimilaron rápidamente el tejido de las escenas de acción y solo en algunos instantes se mostraron a la deriva ante las posibilidades de exploración. Los profesores en estos casos ofrecieron ayuda, pero, al no tener un conocimiento profundo del juego, pidieron a los jugadores más experimentados que explicaran la situación a sus compañeros. Después de finalizar la sesión, los niños hicieron referencia a los niveles del juego,

\subsubsection{Circunstancias personales}

De los once profesores del grupo todos disponen de un ordenador, tableta digital y teléfono móvil. Indican acceder a Internet regularmente desde el ordenador más de dos veces por día. Sin embargo, con el uso de otros dispositivos como tabletas digitales o teléfonos móviles, el número de accesos a Internet se incrementa conectandose más de cinco veces diarias. El número de horas dedicadas al ordenador se resume en cinco horas y dicen dedicarlas a buscar información. El ordenador se utiliza aspectos relacionados con cada profesor: 1) Se pregunto por el uso que hacen de Internet o de otros dispositivos como tabletas digitales, o teléfonos móviles; 2) su formación como docentes y 3) el uso habitual dedicado a los videojuegos fuera del contexto escolar.

centrándose sobre todo en destacar: a) la combinación de elementos y personajes en el "mundo real" y en el "mundo imaginario", b) la narración del juego que conduce a los niños por dinámicas de prueba y repetición que privilegian la dimensión sensorial de la aventura. Los profesores en este sentido resaltaron que los niños enfocaron su atención en las tareas específicas del juego. A continuación se analizan los resultados siguiendo el orden en que se presentaron.

como medio para buscar información y para realizar actividades escolares. El móvil o la tableta se utiliza para comunicarse con los amigos. Sobre su formación como docentes, mencionaron no haber recibido información específica o incentivos para utilizarlos durante su etapa académica universitaria. Manifiestan que en algunos casos de forma parcial se trabajaron algunos aspectos relacionados con las tecnologías, pero esto solo ocurre con los más jóvenes. Sin embargo, 
el profesorado se dedica a autoformarse realizando diversos cursos que incentivan la utilización de herramientas tecnológicas como robots, videojuegos o apps. El informe del Observatori Educació Digital, comprueba que existe un alto nivel de autoformación en Cataluña, siendo el género masculino el que obtiene más representatividad (Coromina, Teberosky y Barbera, 2012). En relación al uso habitual dedicado a los videojuegos

\subsubsection{Actitud}

La actitud de los profesores es un aspecto esencial en el proceso de enseñanza, y sobre todo es básico cuando se aplican juegos digitales en un curso, incluso es necesaria una preparación técnica previa con el fin de resolver los problemas previstos que puedan encontrarse durante el proceso de enseñanza. Khan et al. (2001), mencionan que el profesor debería de tener experiencia y técnica en el tema para proporcionar ayuda a los estudiantes y evitar así un cambio de énfasis en el contenido del curso. Durante la entrevista, los profesores señalaron no tener problemas para entender el juego desde el punto de vista funcional y destacaron la posibilidad de contar con un manual que les describía y ayudaba a contextualizar el juego, innovando metodológicamente en el tratamiento de los contenidos. Sin embargo, la mitad de ellos afirmó tener dificultades para jugar, lo cual no extraña si se atiende al modo en que los videojuegos son una mezcla de ensayo y error, intuición y recepción de fuera del contexto escolar, ningún profesor reconoció ser jugador habitual -de juegos en Internet, teléfono móvil o consola-. Tres de ellos (hombres), mencionaron usar juegos "regularmente", y mencionaron la saga Mario Bros, The Sims o Minecraft. Esta pregunta está centrada en conocer la aceptación y conocimiento del medio fuera del contexto escolar.

información visual. Todos ellos destacan que esto no ocurre con los estudiantes: "Como nativos digitales muestran una gran confianza para manejar cualquier aplicación", "Ellos no se frustran fácilmente". Los 11 entrevistados apreciaron el diseño del juego destacando la información que proporciona, así como la utilidad de contar con un manual de uso. Mencionaron que el tiempo destinado al uso de juegos digitales en el aula no debería ser excesivo: "Hay que controlar la actividad, si no solo se pierde el tiempo", "Los juegos son buenos, pero solo si son dentro de un horario". La actitud es un punto muy importante, porque aunque los profesores no tengan experiencia, el tener una buena disposición permite una continuidad en las actividades del aula. Por ejemplo, durante las sesiones donde fue utilizado el juego, los profesores necesitaron de la ayuda de niños más diestros para explicar a otros alumnos cómo superar las pruebas de habilidad en el juego. Lejos de percibir esto como un problema, lo vieron 
como una oportunidad de trabajar en grupo. De esta forma una debilidad se convirtió en una fortaleza: "Lo que más motiva a los estudiantes es que pueden explicar algo a otro", "Si yo no lo sé, sus compañeros lo explican, se vuelve así una actividad colaborativa". "En una de las sesiones tuvieron que compartir ordenador, pero se divirtieron igualmente porque tuvieron que trabajar en pares". Nueve de ellos indicaron que emplearían el juego Miquel Crusafont en

\subsubsection{Dificultades}

Se menciona, en todos los casos, que la principal dificultad de introduccir un juego de estas características en el aula es que el currículo educativo no contempla un tiempo específico para su uso. Hacen falta, además, tanto metodologías y técnicas activas para que el profesor pueda aplicar juegos en el aula, como modelos que permitan evaluar las habilidades adquiridas por el alumnado. Consideran fundamental conocer todo el potencial de los juegos: "En algunos casos estos juegos no cuentan con un manual de uso, y Crusafont me ha gustado porque muestra claramente cómo puede utilizarse". Destacan que el papel del profesorado es básico a la hora de seleccionar los videojuegos que pueden emplearse en las aulas, y el principal problema detectado es que existen muchos títulos en el mercado, pero pocos de ellos son apropiados para la enseñanza. Ninguno de ellos conocía algun caso similar a Miquel Crusafont con la "capacidad motivadora y un futuro, frente a dos de ellos que dijeron no tener un posicionamiento "claro". Aludieron, básicamente, al temor a tener problemas de gestión en el aula. Otro de los puntos destacados de manera unánime fueron los contenidos y la propuesta de evaluación para desarrollar competencias básicas en matemáticas. Sobre todo señalaron las actividades del juego centradas en interpretar y utilizar números naturales y fraccionarios, de acuerdo con contextos de la vida cotidiana.

componente lúdico". Detectar los apropiados permitiría además recomendar su uso en casa de los escolares. A partir de aquí, se menciona que es necesario motivar a los padres y a las madres para que prueben los mismos juegos que sus hijos e hijas utilizan en las aulas. Respecto a los contenidos presentes en el juego utilizado, todos ellos consideran que son muy útiles. Ven que en la mayoría de productos interactivos educativos no existe una clara relación entre los temas que los alumnos deben trabajar en clase y las ventajas que pueden obtener utilizando esos recursos: "Es necesario tener más información y conocer cuáles son las ventajas de los diferentes juegos que existen y cómo se pueden utilizar". De manera generalizada se pone de manifiesto la posibilidad de no poder controlar el medio: "El problema podría ser que no tenemos control del medio y el no controlarlos puede alejarnos de ellos". 


\subsubsection{Recomendaciones}

La principal recomendación es que se debe desarrollar un clima emocional positivo para el uso de juegos digitales. Por tanto, el profesor en todos los casos debe liderar la iniciativa, este es el principal factor de éxito. Nueve de los profesores indicaron, también, estar de acuerdo en que un juego digital "educativo" debe ser entretenido, pero, además, no debe adaptarse al nivel del usuario. El alumnado es quien, superándose a sí mismo, debe alcanzar el nivel demandado por el juego: "Los niños tienen que superar el nivel de frustración, tienen que equivocarse, porque del error también se aprende". Un total de ocho profesores destacan que, gracias a los juegos, los estudiantes enfocan su atención en las tareas específicas. Valoran, por tanto, el uso de un juego en el aula como una estrategia para poder llegar a más niños, teniendo en cuenta el tiempo que dedican y que estos les motivan: "Cuantas más estrategias o dinámicas tengamos para hacer llegar los contenidos, podremos aprovechar mejor el tiempo y los recursos. También creo que esto depende de la edad y los intereses del alumno, pero los juegos pueden ser positivos y pueden ayudar a trabajar y desarrollar un tema, introducir una parte de este, socializar o entrar en debates a partir de una situación ocurrida en el juego".

Los profesores resaltaron la relación estrecha que se logra entre el alumnado y la narrativa del juego, lo que da pie a que se deriven nuevas historias fuera del juego digital: "Viven el juego y se adentran en él, de hecho después de las pruebas que hicimos en clase con el juego Crusafont, el resto del día los niños estuvieron hablando sin parar del juego y sus personajes". Ya que el juego Miquel Crusafont permite imprimir los personajes del juego, los profesores pudieron utilizar junto con los niños algunos de ellos para crear una extensión del juego virtual. Esta propuesta fue bien recibida y propició nuevas actividades en clase, como la creación de nuevos animales que no figuran en el juego. Recomiendan que otras propuestas repliquen esta misma idea pero con el uso de manuales. Finalmente, resaltan que el videojuego genera una mayor motivación que cualquier otro producto interactivo. Al ser el "videojuego" un producto más cercano al alumnado, su uso en el aula es una buena estrategia para desarrollar competencias. Se incentiva así el aprendizaje basado en estrategias cognitivistas y sirve como estimulación para lograr un proceso de mejora en los resultados. Manifestaron además ser conscientes de que, gracias a un juego como Miquel Crusafont, los alumnos adquieren competencias que son importantes para su futuro, fuera de la formación reglada: "Prima no solo el aporte metodológico, como recurso, sino también los contenidos específicos que incluye y su tratamiento didáctico para la enseñanza de las matemáticas o la lengua, así como 
la cultura y aspectos relacionados con la vida cotidiana". En concreto, nueve de ellos consideraron que a través de los videojuegos se puede trabajar cualquier contenido propio de esta etapa educativa, mientras que dos de ellos opinan que solo es posible trabajar con contenidos de corte matemático y mostrar contenido para las ciencias de la

\section{Conclusiones}

El propósito de nuestra evaluación fue analizar y valorar la experiencia obtenida por los profesores de primaria, utilizando un juego digital como Miquel Crusafont. Los profesores utilizan regularmente diversos dispositivos como medios para buscar información, realizar actividades escolares o para socializar con amigos y familiares. Significa que están habituados al uso de dispositivos. Sobre su formación como docentes, a pesar de que no recibieron información específica o alicientes para utilizar juegos digitales o videojuegos en las aulas, se dedican a autoformarse realizando cursos que incentivan su utilización. Y aunque no todos hacen un uso habitual de estos medios fuera del contexto escolar, aceptan y valoran la utilización de juegos digitales en las aulas. Como hemos mencionado anteriormente, su actitud es un aspecto esencial en el proceso de enseñanza, y en este sentido los profesores demostraron una actitud positiva tanto para entender el juego como en su aplicación en las aulas. No percibieron que el alumnado tuviera problemas destacables para utilizar el juego de Crusafont, aunque ellos sí tuvieran naturaleza. En el caso de las ciencias sociales, nueve de ellos hacen especial mención a los contenidos que pueden introducirse en un videojuego, como el conocimiento respecto a diferentes culturas, el concepto de ciudad, las nociones económicas y espaciales, así como procedimientos de seriación, clasificación y observación.

dificultades. Destacan incluso que en la mayoría de actividades interactivas ocurre lo mismo, los escolares son mucho más hábiles. La actitud positiva del profesorado permite favorecer una continuidad en las actividades del aula y una oportunidad para descubrir nuevas metodologías de aprendizaje, sin detectar un esfuerzo extra de trabajo. Apreciaron además el diseño del juego centrado en la figura de un paleontólogo destacando los contenidos que proporciona, centrados en el currículo, así como la utilidad de contar con una guía clara para su uso. Incluso las actividades propuestas para evaluar actividades. La opinión mayoritaria es "la necesidad de controlar la actividad dentro de un horario". Lo que significa que dentro de este escenario novedoso que facilita desarrollar competencias y destrezas es necesario establecer unos límites. También indicaron que emplearían el juego Crusafont en un futuro, ya que lo ven como una herramienta de utilidad para las aulas. Las principales dificultades detectadas desde el punto de vista de los profesores de primaria 
para introduccir un juego de las características de Miquel Crusafont en el aula son: a) falta un tiempo específico en el currículo destinado al uso de juegos digitales, b) hacen falta metodologías y técnicas activas para que el profesor pueda aplicar juegos en el aula; c) faltan modelos que permitan evaluar las habilidades adquiridas por el alumnado; c) es necesario además motivar a los padres, madres y tutores o tutoras para que utilicen los mismos juegos digitales que sus hijos o hijas utilizan en las aulas.

En cuanto a las recomendaciones, el profesorado indica: a) se debe desarrollar un clima emocional positivo en el aula para el uso de juegos digitales; b) deben ser los profesores, en todos los casos, quienes deben liderar la iniciativa; c) los juegos educativos utilizados en el aula deben ser entretenidos y adaptarse al nivel del usuario permitiendo así una superación personal; d) los juegos deben incluir diversas estrategias y dinámicas para hacer llegar los contenidos; e) llevar la experiencia educativa más allá del juego virtual; f) los juegos deben de contar con un manual de instrucciones que muestre toda la potencialidad del juego.

La conclusión general es que una vez que los educadores observaron el comportamiento de los alumnos en las dos sesiones de juego, pudieron aprecian la potencialidad de un juego de las caracteristicas de Miquel Crusafont para desarrollar competencias en áreas como las matemáticas, las lenguas o las ciencias de la naturaleza. Vieron, además, cómo pueden alentar a los estudiantes a realizar actividades de aprendizaje colaborativo y contextualizar actividades en base al juego digital.

Creemos que la idea de generar una experiencia didáctica como esta va en correcta alineación con la vía de integración de las TIC en el currículo de primaria, a pesar de que dicha integración no dependa ni única ni fundamentalmente de una toma de decisiones en cuanto a la implementación de una metodología. El análisis desde la perspectiva del profesorado ha puesto en evidencia algunas de las razones que pueden parecer difíciles de controlar y que demuestran la situación del tema. Esta experiencia revela unas circunstancias que concuerdan con algunas de las investigaciones detectadas por Perrota et al. (2013) y que hacen patente la dificultad de enraizar el ABJ o el uso de los JS en las prácticas educativas. Lamentablemente, el currículo oficial no está adaptado, la organización de espacios y tiempos en las escuelas, así como los hábitos socioculturales, son factores contribuyentes a que el proceso sea lento y difícil, y aun en el supuesto de que los diseños tecnopedagógicos teóricamente eficaces o fundamentados en planteamientos de enseñanza-aprendizaje fueran ampliamente aceptados, faltaría que estos fueran validados por la entidades correspondientes. El futuro nos invita a seguir estudiando e implementando nuevas experiencias y replanteando las razones por las que el ABJ y los JS todavía no cuentan con la capacidad suficiente de transformar la dinámica educativa en el nivel de primaria. 


\section{Referencias}

Bonk, C., \& Dennen, V. (2005). Massive multiplayer online gaming a research Kearney, C. (2011). European Schoolnet's framework for military training and Games in Schools Study: The Current education. Washington, D.C.: Office of the State of Play in European Schools and the Under Secretary of Defense (Personnel Game Ahead. In F. Patrick (Ed.), Handbook and Readiness), Readiness and Training of Research on Improving Learning and Directorat, Advanced Distributed Learning Motivation through Educational Games: (ADL) Initiative. http://dx.doi.org/10.1037/ Multidisciplinary Approaches (pp. 51e448112006-001

Coromina, J., Teberosky, A., \& Barberà, E. (2012, July 1). L'Ús de les eines digitals 2.0 en L'educació primària a Barcelona. Retrieved January 13, 2015, from http:// openaccess.uoc.edu / webapps / o 2 / bitstream/10609/31141/1/OED_Informe_ eines_digitals_20_educacio_primaria-1.pdf

Flavell, J. (1983). Cognitive Development (4th ed.). New York: Wiley.

Fuller, M. \& Jenkings, H. (1995). Nintendo and New World Travel Writing: a Dialogue. Thousand Oaks

Sage Publications.

Higgins, E., Grant, H., \& Shah, J. (1999). Self Regulation and quality of life: Emotional and nonemotional life experiences. In D. Kahneman, E. Diener, \& N. Schwarz (Eds.), Well-being: The foundations of hedonic psychology (pp. 244-266). New York: Russell j.1467-8535.2009.01007.x

Sage Foundation.
73). Hershey: IGI Global. http://dx.doi. org/10.4018/978-1-60960-495-0.ch003

Khan, K., Riet, G., Glanville, J., Sowden, A., \& Kleijnen, J. (2001). Undertaking systematic reviews of research on effectiveness: CRD's guidance for those carrying out or commissioning reviews (2nd ed.). York, England: Centre for Reviews and Dissemination, University of York.

Kirriemuir, J., \& McFarlane, A. (2004, January 1). Literature review in games and learning. Retrieved May 2, 2010, from http://www.futurelab.org.uk/download/ pdfs/research/lit_reviews/Games_Review1

Kenny, R., \& McDaniel, R. (2011). The role teachers' expectations and value assessments of video games play in their adopting and integrating them into their classrooms. British Journal of Educational Technology, 42(2), 197-213. http://dx.doi.org/10.1111/ 
Mitchell, A., \& Savill-Smith, C. (2004). The dx.doi.org/10.1016/0361-476X(83)90018-8 use of computer and video games for learning: A review of the literature. London: Learning Vygotsky, L. S. (1978). Mind in Society. and Skills Development Agency. Cambridge: Harvard University Press.

Perrotta, C., Featherstone, G., Aston, H., \& Wu, B., \& Wang, A. (2012). A Guideline Houghton, E. (2013). Games-based Learning: for Game Development-Based Learning: A Latest Evidence and Future Directions. Literature Review. International Journal of Slough: NFER. Computer Games Technology, 1-20. Retrieved February 5, 2015, from http://www.hindawi.

Paris, S., Lipson, M., \& Wixson, K. (1983). com/journals/ijcgt/2012/103710 http://

Becoming a Strategic Reader. Contemporary dx.doi.org/10.1155/2012/494232

Educational Psychology, 8, 293-316. http:// 